\title{
iMatTOUGH: An Open-source Matlab-based Graphical User Interface for Pre- and Post- Processing of TOUGH2 and iTOUGH2 Models
}

\author{
Anh Phuong Tran, Baptiste Dafflon, Susan Hubbard \\ Lawrence Berkeley National Laboratory \\ Earth Sciences Division \\ Berkeley, CA, 94720, USA \\ E-mail: APTran@lbl.gov
}

\section{ABSTRACT}

TOUGH2 and iTOUGH2 are powerful models that simulate the heat and fluid flows in porous and fracture media, and perform parameter estimation, sensitivity analysis and uncertainty propagation analysis. However, setting up the input files is not only tedious, but error prone, and processing output files is time consuming. In this study, we present an open source Matlab-based tool (iMatTOUGH) that supports the generation of all necessary inputs for both TOUGH2 and iTOUGH2 and visualize their outputs. The tool links the inputs of TOUGH2 and iTOUGH2, making sure the two input files are consistent. It supports the generation of rectangular computational mesh, i.e., it automatically generates the elements and connections as well as their properties as required by TOUGH2. The tool also allows the specification of initial and timedependent boundary conditions for better subsurface heat and water flow simulations. The effectiveness of the tool is illustrated by an example that uses TOUGH2 and iTOUGH2 to estimate soil hydrological and thermal properties from soil temperature data and simulate the heat and water flows at the Rifle site in Colorado. 


\section{OVERVIEW}

18 TOUGH2 is a nonisothermal multi-phase, multi-component model that simulates the heat and fluid flows in porous and fractured media (Pruess et al., 1999). iTOUGH2 is a program that is used for parameter estimation, sensitivity analysis and uncertainty propagation analysis for TOUGH2 (Finsterle, 1999). TOUGH2 and iTOUGH2 have more and more caught the attention of the scientific community and been successfully applied in various research disciplines, namely, carbon storage, environmental remediation, hydrology, geothermal, landfill, nuclear TOUGH2 and 56 publications using iTOUGH2 difficulties in pre-processing inputs and post-processing outputs have limited their popularity as they should be. Although the input files in TOUGH2 and iTOUGH2 are well organized and structured, the natural complexity of these models and the formatted, text-based input files are error prone and time-consuming for users to manage. Moreover, it is not convenient for users to graphically analyze the outputs of either model with their text format. For example, it takes a measured and modeled data is also not straightforward. To remedy these limitations, we develop iMatTOUGH. 
Prior to the development of iMatTOUGH, several GUIs supporting TOUGH2 have been developed. For example, Li et al. (2011) developed TOUGHER to control the input and output of TOUGHREACT. Adrian Croucher (University of Auckland, New Zealand) developed a library of Python scripts (named PyTOUGH) for automating setup and execution of TOUGH simulations. Using PyTOUGH, Wellmann et al. (2012) developed a workflow that processes grid generation, model setup, execution, and result analysis. Berry et al. (2014) developed TOUGH2GIS, a GIS-based pre-processor that allows the user to create locally refined unstructured (Voronoi) grids and to assign rock types to grid blocks. In addition to these opensource programs, there are commercial products that support the development of TOUGH2 models, e.g. PetraSim (http://www.thunderheadeng.com/petrasim/), mView (http://geofirma.com/software/mview/), and Leapfrog (http://www.leapfrog3d.com/).

However, no program exists to simultaneously process the inputs and outputs of TOUGH2 and iTOUGH2 as well as the linkage between them. In this study, we present iMatTOUGH, a Matlab-based tool that allows users to generate all necessary inputs for both TOUGH2 and iTOUGH2, execute these models, and visualize and analyze their outputs. The idea of development of this tool is to hide the complicated input and output data of TOUGH2 and iTOUGH2 from users, allowing them to concentrate on analyzing model results. In addition, iMatTOUGH is capable of creating the rectangular computational mesh. Elements and connections as well as their associated properties (e.g., element volumes, element materials, interface areas at connections, etc.) are internally generated by iMatTOUGH. The upper and lower boundary elements are automatically defined. Particularly, the tool supports users to process the initial and time-dependent boundary conditions for a better simulation of subsurface heat and water flows, which is usually tedious and causes a lot of errors for users. The upper 
pressure boundary condition is adjusted to account for its dependence on the elevation of the study site. The upper air mass fraction boundary condition is calculated from the atmospheric temperature and humidity data. Instead of the atmospheric temperature, the upper temperature boundary condition is the soil surface temperature, which is estimated from the atmospheric temperature using the atmospheric - soil surface temperature regression equation at a reference location with consideration of snowpack. Rainfall is processed so that it can be considered as source flux Neumann boundary condition at the soil surface elements. The bottom pressure boundary condition is estimated from the groundwater level, while the bottom temperature boundary condition is approximated using the analytical solution of heat transport equation. Finally, the initial conditions are estimated from the measurement data if they are available at the beginning of the simulation period.

\section{PROGRAM STRUCTURE AND GRAPHICAL INTERFACE}

iMatTOUGH is an open-source code written in Matlab, which is sufficiently flexible for users to update/modify the code for their specific purposes. The code can be downloaded at https://www.dropbox.com/sh/n3yo418puv6ocax/AADTAixV6MS1uH3w0u_541LVa?dl=0.

Figure 1 presents the iMatTOUGH structure. The program includes four main components that allow users to generate TOUGH2 and iTOUGH2 input files (pre-processing), execute them and visualize their results (post-processing). The detailed information about these components are presented as below:

\subsection{Main Control}


Figure 2 presents the main control GUI, which is responsible for calling all pre- and post-

87 processing programs, namely, 1) generate the TOUGH2 input file, 2) generate the iTOUGH2

88

89

90

91

92

93

94

95

96

97

98

99 input file for inversion, 3) generate the iTOUGH2 input file for sensitivity analysis, 4) visualize the TOUGH2 output file and 5) visualize the iTOUGH2 output file. Because iMatTOUGH uses some information from the TOUGH2 input file (e.g., names of materials, elements and connections) to generate the iTOUGH2 input, the TOUGH2 input file must be created first. One typical characteristic of the program is its context-based approach, i.e., when possible, the program automatically fills information for users to reduce their workload and mistakes. For example, if users select to perform a sensitivity analysis, the program will show only requirements related to sensitivity analysis and hide all requirements for parameter estimation. If the observation variable is temperature/pressure/saturation, the program will understand that it is observed at an element and shows only information related to the elements. By contrast, if the observation is flow rate, the program will present the requirements for connections. This contextbased approach is applied for both pre- and post-processing. 


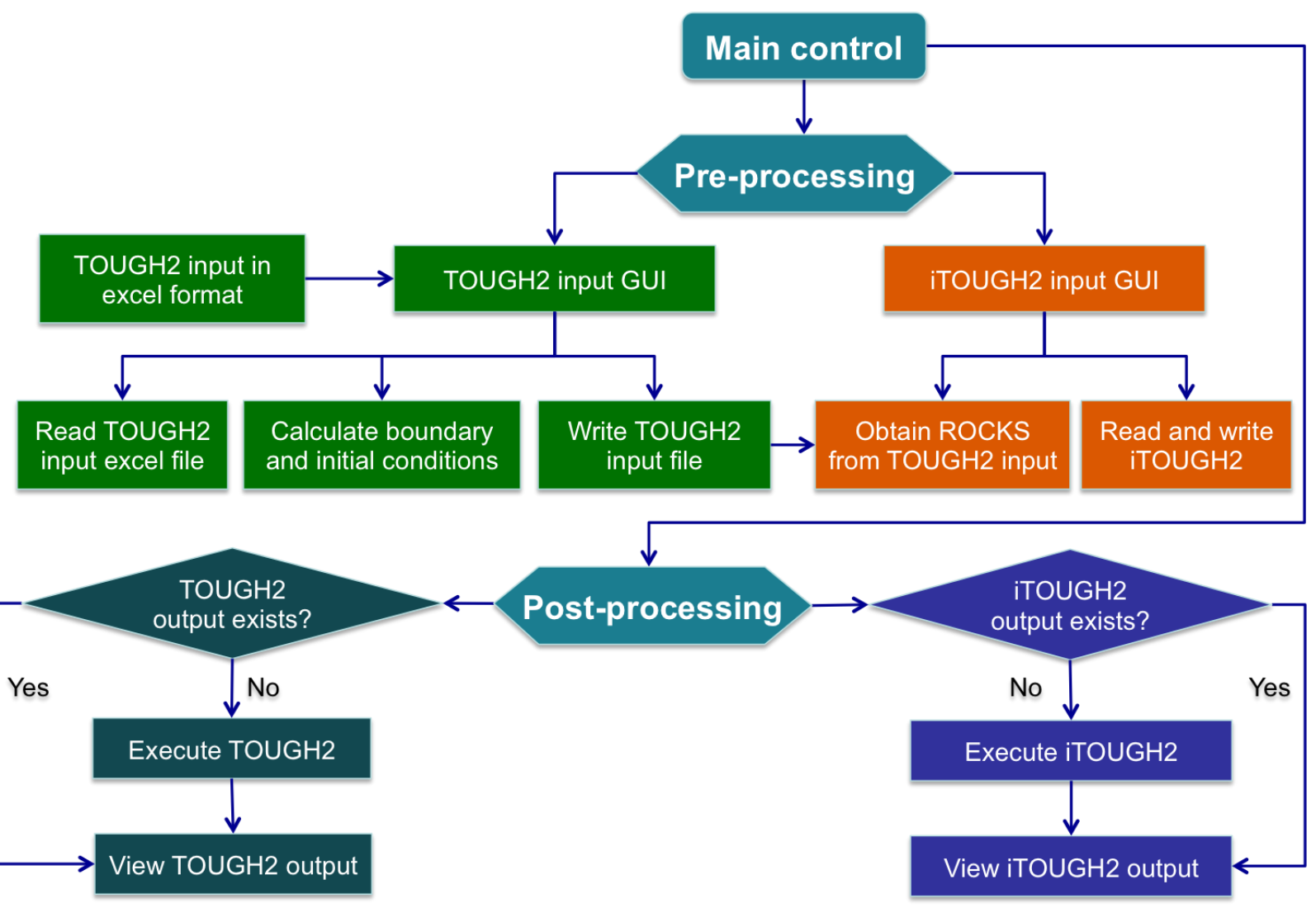

101 Figure 1. Workflow of the iMatTOUGH for pre- and post-processing TOUGH2 and iTOUGH2

102 models

103

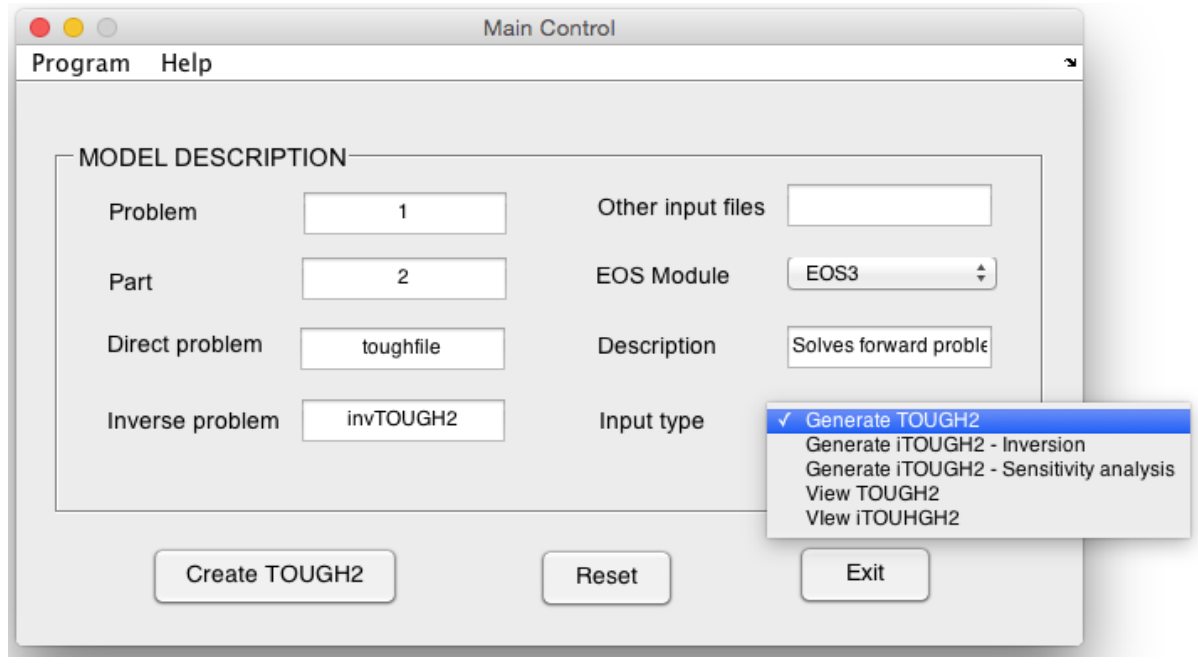

Figure 2. Main control interface 


\subsection{Pre-processing}

\subsubsection{Pre-processing TOUGH2 input file}

Input data for TOUGH2 are provided by one or several ASCII files and organized in blocks for different types of inputs (e.g., soil materials, mesh elements, mesh connections, initial and boundary conditions, simulation and printout control parameters). Each block begins with keywords that are defined by TOUGH2. The main difficulty when editing the TOUGH2 input file is that users must strictly follow a stringent input format (e.g., keyword names, number of spaces for each input, order of the input blocks). iMatTOUGH supports users by automatically formatting the input data as required by TOUGH2. Figure 3 shows the GUI for TOUGH2 input generation. Users only need to provide required information for TOUGH2 in an EXCEL file as shown in Figure 4. At the TOUGH2 GUI, users must identify the location of the TOUGH2 input EXCEL file, the beginning and end of the simulation period and the option selected for the computational mesh. There are 3 options for the mesh generation including iMatTOUGHMeshMaker (mesh is generated by iMatTOUGH), User input (users provide information for elements and connections in EXCEL file) and TOUGH2-MeshMaker (mesh is generated by TOUGH2). The EXCEL file includes 15 sheets corresponding to different blocks in TOUGH2 input file and additional information for rectangular mesh generation (iMatTOUGHMeshMaker), and specifying boundary (BOUND) and initial (INITIAL) conditions for shallow subsurface simulation. The program will read the EXCEL file, process initial and boundary conditions (if necessary) and write the TOUGH2 input file. In order to help iMatTOUGH read the TOUGH2 input data, users should use the template EXCEL file to put their data. 


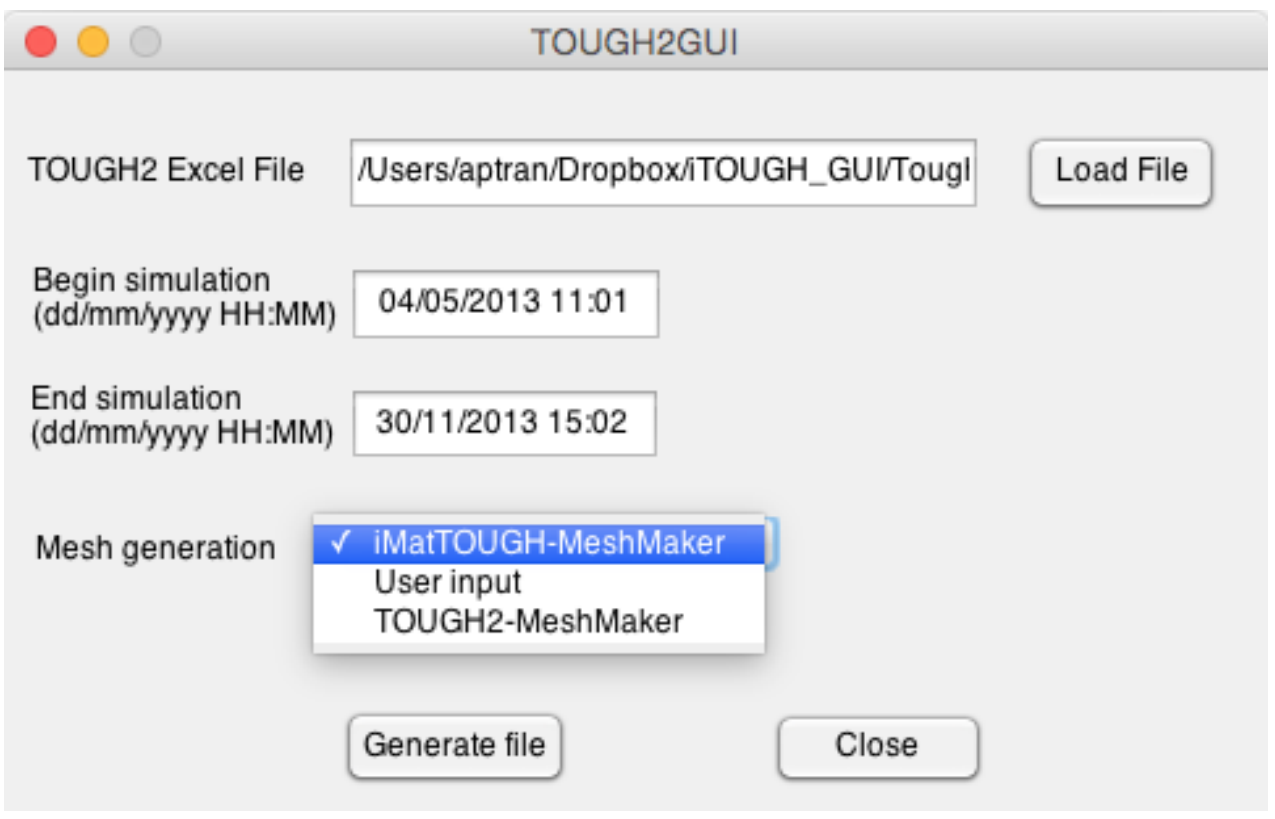

Figure 3. User interface for TOUGH2 input file generation.

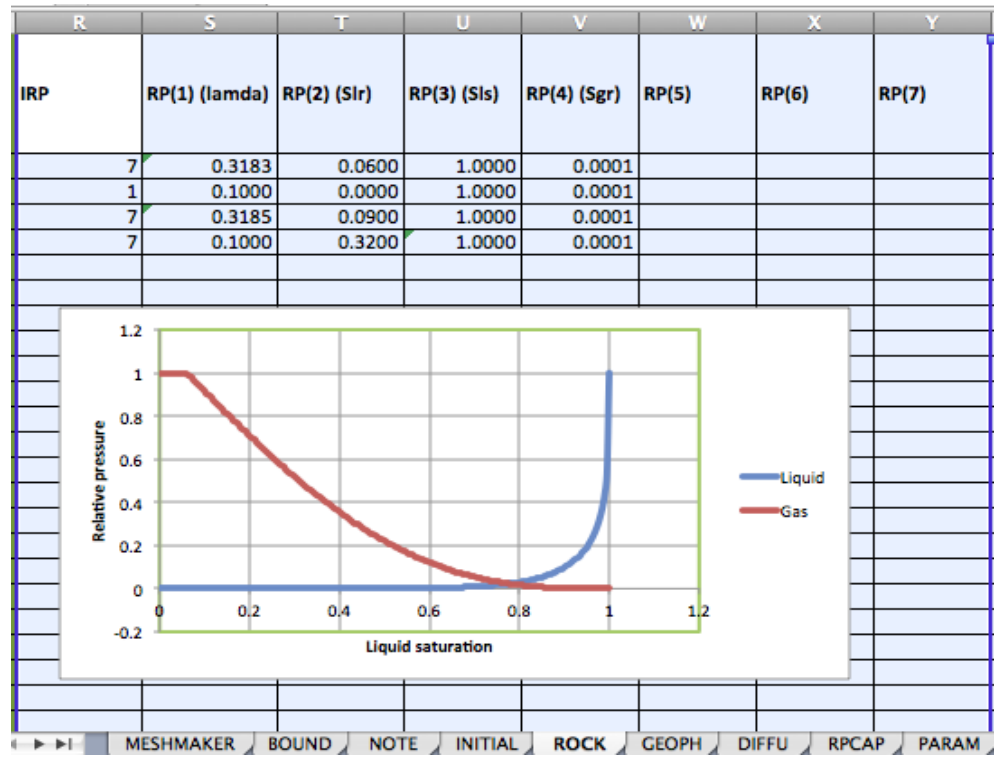

134 Figure 4. Example from the TOUGH2 EXCEL spreadsheet, showing the input of relative permeability function parameters. 
The iTOUGH2 input is also an ASCII file. However, instead of using formatted input, it employs

140 a high-level input language for convenient specification of information in free format. The file

141 contains commands that are structured hierarchically. Each command belongs to a parent

142 command and may contain one or more child commands on a lower command level. Compared to the TOUGH2 input file, it is more flexible to create the iTOUGH2 file. However, users still

144 have to follow the regulations of the command names and command structures. Our program provides a list of available iTOUGH2 commands for users to select, and it internally structures the input file as required by iTOUGH2. Figure 5 presents the main GUI for iTOUGH2 input generation and its child windows. The main interface is, which appears when users select "Generate iTOUGH2 - inversion" or "Generate iTOUGH2 - sensitivity analysis" options in Figure 2, divided into three sections, corresponding to the three highest command levels in the iTOUGH2 input file, namely, PARAMETER, OBSERVATION and COMPUTATION. The PARAMETER section requires information about the parameters to be varied for sensitivity analysis, parameter estimation, or uncertainty propagation analysis (e.g., parameter name, its initial guess, range and variation). The OBSERVATION section requires information related to 154 observable variable and their corresponding measured data. When users click the "Add" button, a child widow as shown in Figure $5 \mathrm{~b}$ is opened to help users to provide detailed information of a 156 given data type (e.g., locations of measurements, time unit, data filename, observation standard deviation). The COMPUTATION section requires information on program options and computational control parameters (e.g., optimization algorithm, sensitivity analysis method, type of objective function, convergence criteria, output options, etc.). These requirements are inputted in 4 child windows corresponding to 4 child commands of the COMPUTATION, namely, error, 161 stop, option and output as shown Figure 5c, d, e and f. Figure 5b appears when users click "Add" 162 button in the OBSEVATION section in Figure 5a. Figures 5c, d, e and $\mathrm{f}$ appear when users, 
163 respectively, select "Error", "Stop", "Option" and "Output", and then, click "Edit" button in the 164 COMPUTATION section. For more detailed information about these requirements, we refer to 165 Finsterle (1999). Before opening the GUI for editing the iTOUGH2 input, the program loads the 166 TOUGH2 input file and populates the soil material popup box in the PARAMETER section, and

167 the element and/or connection popup boxes in the OBSERVATION section. This helps users to 168 assure consistency between the TOUGH2 and iTOUGH2 inputs.

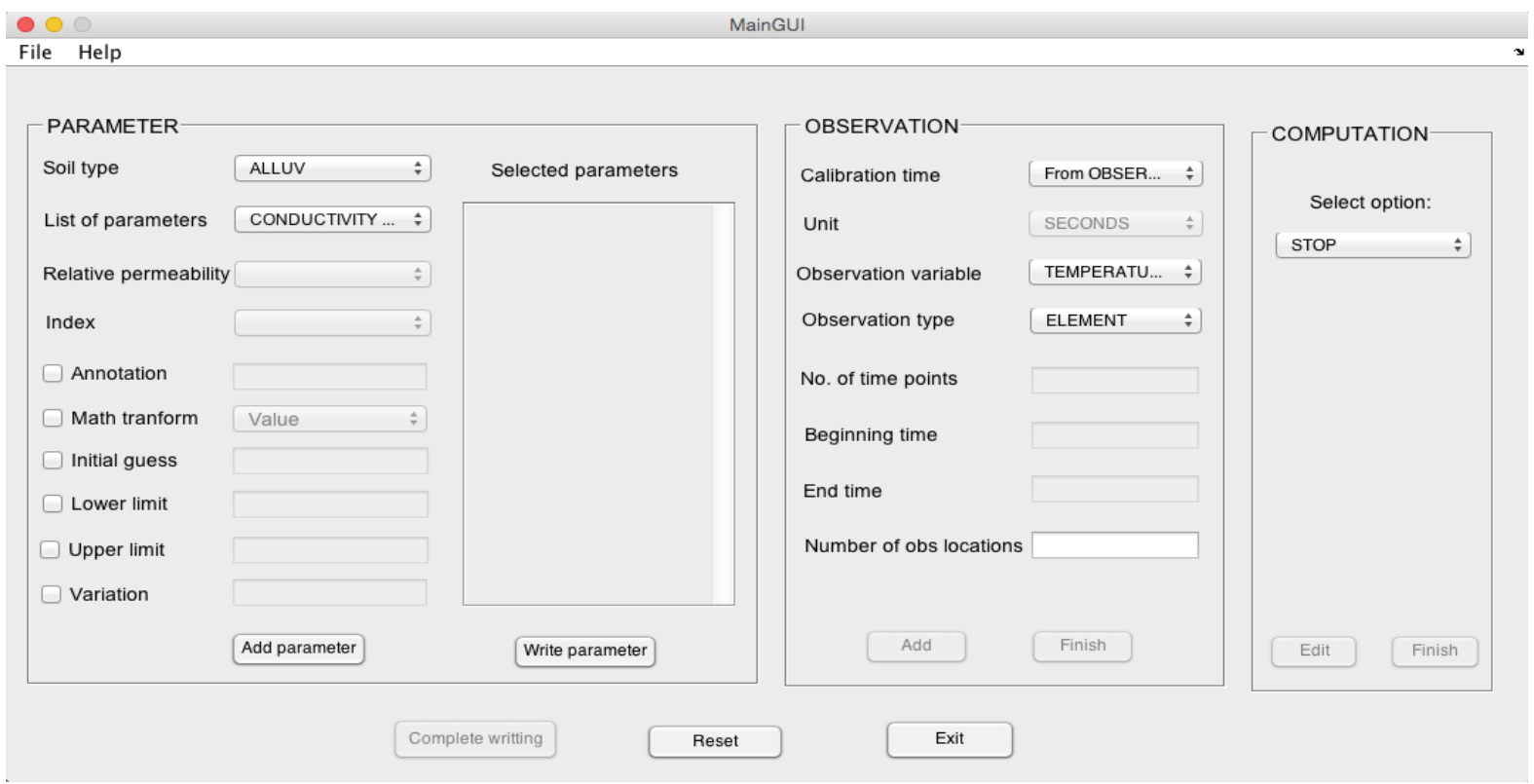

(a) 


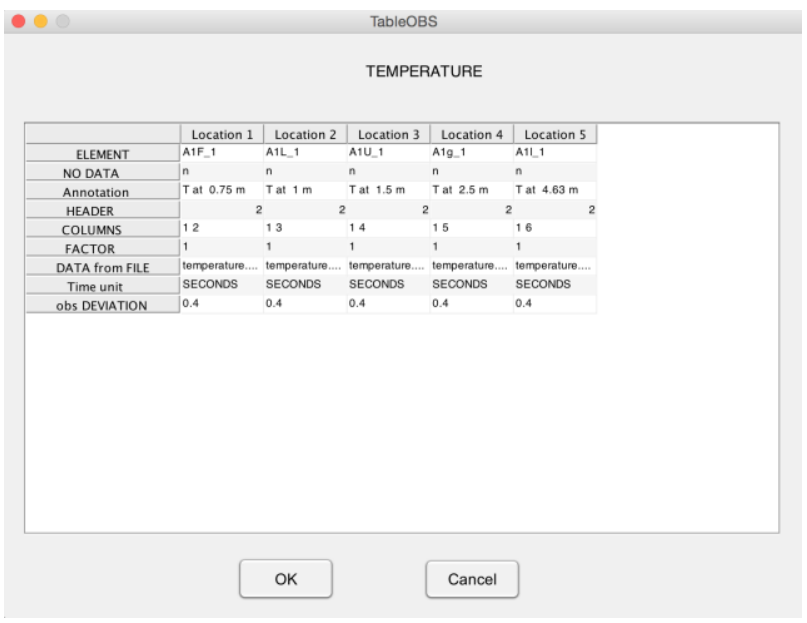

(b)

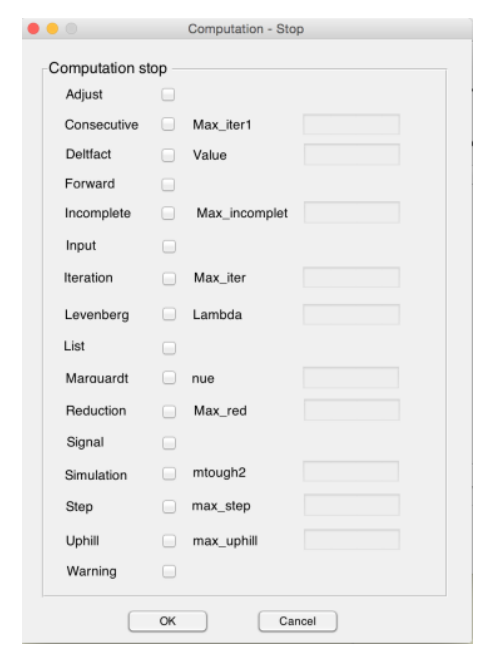

(d)

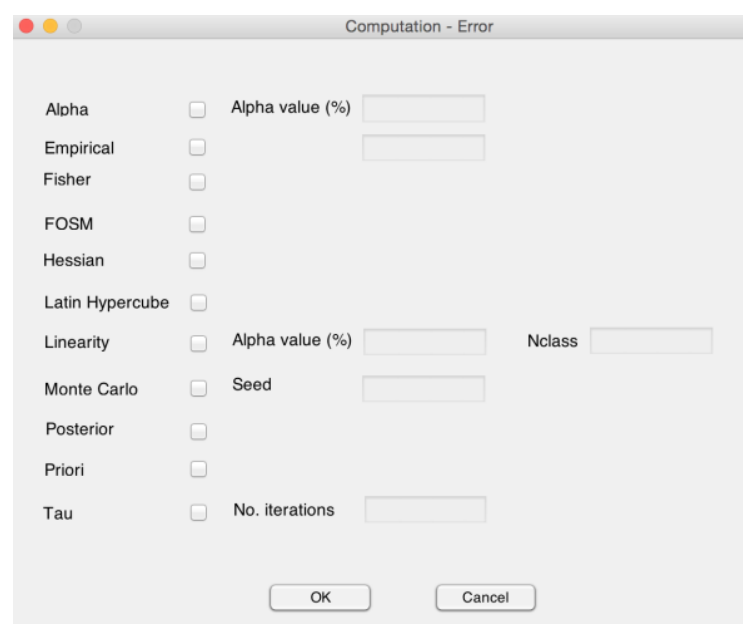

(c)

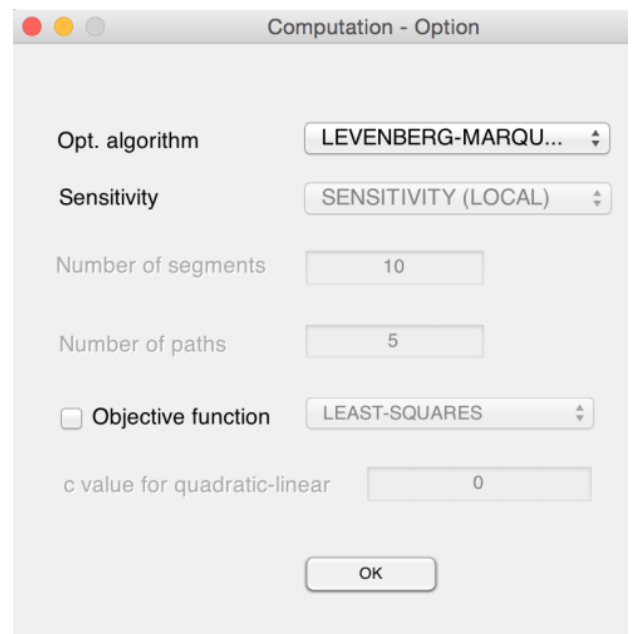

(e)

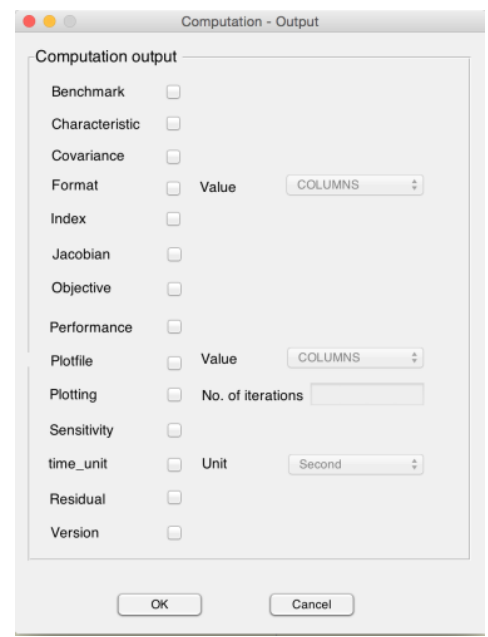

(f)

Figure 5. Main user interface for iTOUGH2 input generation (a) and its child windows for providing observation information (b) and information on program options and computational control parameters including error (c), stop (d), option (e) and output (f). Figure 5b appears when users click "Add" button in the OBSEVATION section in Figure 5a. Figures 5c, d, e and f appear when users respectively select "Error", "Stop", "Option" and "Output", and then click "Edit" button in the COMPUTATION section. 


\subsubsection{Mesh generation}

One of the hardest pre-processing tasks is to generate the computational mesh, which is composed of elements and connections between these elements. If the computational mesh data are available (e.g., from output of a mesh generation code), users can provide these data in the EXCEL input file (types of soil/rock materials are putted to the ROCK sheet; elements and connections between elements data are putted to the ELEME sheet). In case the element and connection data as well as their associated information (e.g., material type, volume, distance to interface, interface area) are not available, iMatTOUGH can support users to create the computational mesh. In the current version of iMatTOUGH, the tool can only create the rectangular mesh. To do that, users need to provide the number of grid cell increments and grid sizes in $\mathrm{X}, \mathrm{Y}$, and $\mathrm{Z}$ directions. It automatically sets the name of elements, internally makes the connections between them as well as determines the boundary elements of the computational domain. In addition, if users provide the spatial ranges of the soil/rock types (e.g., clay soil in range $4<\mathrm{X}<5 \mathrm{~m}, 0<\mathrm{Y}<1 \mathrm{~m},-1.8<\mathrm{Z}<0 \mathrm{~m})$, the program will automatically assign the soil/rock types to elements based on these ranges and the element coordinates.

\subsubsection{Initial and boundary conditions for shallow subsurface simulation}

If the data for initial and boundary conditions are available, they can be provided in the EXCEL file in Figure 4 to write into the TOUGH2 input file. This section presents an add-in tool that pre-processes the initial and boundary conditions for the cases of nonisothermal shallow subsurface heat and water flow simulation. The calculation of these initial and boundary conditions are presented below: 
204 Upper pressure boundary condition: The upper pressure boundary condition is the atmospheric 205 pressure, which is adjusted from the standard atmospheric pressure with consideration of the 206 elevation as:

$$
P_{a}=P_{0}\left(1-\frac{L h}{T_{0}}\right)^{\frac{g M}{R L}}
$$

Upper temperature boundary condition: Because the current version of TOUGH2 cannot simulate the radiation and energy exchanges at the soil surface, the upper temperature boundary condition should be the soil surface temperature instead of the atmospheric temperature. If soil surface temperature data are not available, they can be estimated from the atmospheric temperature and snowpack data based on the historical data of the atmospheric and soil surface temperatures at a nearby station (reference location) following the approach introduced in Zheng et al. (1993):

1. Calculate $n$-day running averages of daily air temperature to smooth fluctuations. The number of days for the running average is determined so that the estimated and measured soil surface temperature data are best fit. Next, establish the linear regression equation between the smooth air temperature with the daily soil surface temperature (without smoothing) at the reference location where both air and surface temperature data are available. 
2. Use the regression equation in Step 1 to estimate the daily soil surface temperature $\left(T_{r}\right)$ at the location of interest.

3. Adjust $T_{r}$ obtained in Step 2 based on the presence of snowpack on the soil surface. If snowpack exists, the soil surface temperature is temporally more stable, and thus, the soil surface temperature $T_{s}^{i}$ at date $i$ is more closely linked to $T_{r}^{i-1}$ at the previous date $i-1$ and the equation below is used:

$$
T_{s}^{i}=\left(T_{a}^{i}-T_{a}^{i-1}\right) M_{1}+T_{r}^{i-1}
$$

When snowpack is not present, the soil surface temperature is more closely linked to $T_{r}^{i}$ and the equation below is used:

$$
T_{s}^{i}=\left(T_{a}^{i}-T_{a}^{i-1}\right) M_{2}+T_{r}^{i}
$$

$T_{a}$ represents the daily air temperature; and $M_{1}$ and $M_{2}$ are the empirical coefficients which were determined by Zheng et al. (1993) as $M_{1}=0.1$ and $M_{2}=0.25$.

Upper air mass fraction boundary condition: The upper boundary condition for the air mass fraction is calculated from the atmospheric temperature and humidity data as suggested by the National Oceanic and Atmospheric Administration (http://www.srh.noaa.gov/epz/?n=wxcalc_vaporpressure):

$$
e_{s}=6.11 \times 10^{\frac{7.5 T_{a}}{237.3+T_{a}}}
$$

$$
e_{a}=\frac{R H}{100} e_{s}
$$

$$
X_{v}=\frac{0.018 e_{a}}{0.029 P_{a}}
$$

$$
X_{a}=1-X_{v}
$$


248 in which $e_{s}$ and $e_{a}$ are, respectively, the saturated and actual vapor pressure, respectively; $X_{v}$ and $249 X_{a}$ denote the mass fraction of water vapor and air; and $R H$ is the atmospheric relative humidity.

Bottom pressure boundary condition: If the bottom boundary is below the groundwater table, the

252 bottom pressure boundary condition can be calculated from the groundwater table depth as:

$$
P=P_{a}+\rho g\left(z-z_{W L}\right)
$$

in which $z$ and $z_{W L}$ are, respectively, the depth of the bottom boundary and groundwater table; $\rho$ is the density of water.

Bottom temperature boundary condition: The bottom temperature boundary condition is approximated from its nearest location where the measured temperature data are available (reference location) using the analytical solution of the heat transport equation, assuming that the temporal variation of the temperature is sinusoidal (van Wijk and de Vries, 1963):

$$
T(z, t)=\bar{T}+A_{s} e^{-\frac{z}{d}} \sin \left(\frac{2 \pi t}{\tau}-\frac{z}{d}\right)
$$

in which $\bar{T}$ and $A_{s}$ are the mean and amplitude of the temperature at the reference location; $\tau$ is the period, e.g., if the annual variation is considered, $\tau=1$ year; $z$ is the distance between the reference location and the bottom boundary; $t$ is the relative time with respect to time $t_{0}$ at which the temperature at the reference location is equal to $\bar{T} ; d$ is the damping depth which is defined as:

$$
d=\sqrt{\frac{\tau D}{\pi}}
$$

in which $D$ is the thermal diffusivity which relates to the thermal conductivity $(\lambda)$ and heat capacity $\left(C_{R}\right)$ by $D=\lambda / \rho_{\text {bulk }} C_{R}$ in which $\rho_{\text {bulk }}$ is the bulk density of the soil mixture including soil matrix, air and water content. $\lambda, \rho_{\text {bulk }}$ and $C_{R}$ are properties of the soil between the reference 
271 location and the bottom boundary. In iMatTOUGH, users need to provide parameters $\bar{T}, A_{s}, \tau$ 272 and $D$. In case the measured soil temperature data are not available, the bottom temperature 273 boundary condition can be obtained from the estimated soil surface temperature with the 274 reference location at the soil surface.

Rainfall: Rainfall can be considered as water sources at all surface elements in TOUGH2. Due to difference in units between the rainfall data and TOUGH2 source/sink $(\mathrm{kg} / \mathrm{s})$, the unit conversion 278 from rainfall to source unit is performed as follows:

$$
S_{\text {TOUGH2 }}^{i}=C R_{a} A^{i} \rho
$$

in which $C$ is the unit conversion coefficient from measured rainfall unit to $\mathrm{m} / \mathrm{s}$, e.g., if rainfall

281 unit is inch/hour, $C=\frac{0.0254}{3600} ; R_{a}$ is the rainfall; $\rho$ is the density of rain water $\left(\rho=1000 \mathrm{~kg} / \mathrm{m}^{3}\right)$;

$282 S_{\text {TOUGH2 }}^{i}$ is the rainfall-equivalent source rate in TOUGH2 at the surface element the element $283 i^{t h}$ and $A^{i}$ is the horizontal interface area between the atmospheric layer and the element $i^{\text {th }}$ $284\left(m^{2}\right)$ of the element $i^{\text {th }}$ at the soil surface where rainfall arrives.

It is worth noting that all of the above boundary conditions are time-dependent conditions. 287 Except for the rainfall is considered as sources and the Neumann boundary condition, the other 288 data are Dirichlet boundary conditions, which are not available in TOUGH2 but supplemented in 289 iTOUGH2.

Initial conditions: Initial conditions of the whole computational domain (pressure, gas saturation 292 (or air mass fraction) and temperature) are required to start the TOUGH2 simulation. The initial 293 pressure at the atmosphere layer and unsaturated zone are set to the atmosphere pressure 294 (Equation (1)). The initial pressure at the saturated zone is determined based on the relative depth 
295 of the grid cell with respect to the initial groundwater table depth using Equation (8). The initial

296 subsurface temperature profile is estimated by interpolating and extrapolating the temperature

297 data measured at different depths at the beginning of the simulation period. In case that the soil

298 temperature measurements are not available, the initial soil moisture profile is approximated

299 from the soil surface temperature using Equation (9). The initial value of the air mass fraction of

300 the single-phase gas at the atmospheric layer is calculated using Equations from (4) to (7). If the

301 matric potential measurements are available, the initial condition of the gas saturation of the

302 unsaturated zone can be estimated as follows:

303 1. Convert initial values of the measured matric potential at the beginning computation time $\left(P_{c}\right)$ to the liquid saturation $\left(S_{l}\right)$ by using the retention curve function, e.g., the van Genuchten's equation (van Genuchten, 1980) below:

$$
S_{l}=S_{l r}+\left(S_{l s}-S_{l r}\right)\left[1+\left(-P_{c} \alpha\right)^{\frac{1}{1-m}}\right]^{-m}
$$

2. Calculate the gas saturation $S_{g}=1-S_{l}$;

\subsection{Post-processing}

3. Interpolate gas saturation to all unsaturated grid cells of the computational domain.

\subsubsection{TOUGH2 viewer}

317 TOUGH2 prints out all of its output variables at each time step. This aspect renders it time- 
TOUGH2 results. Before showing this GUI, the program first checks if the TOUGH2 output file

320 is available. If the output file is not available, the program will execute TOUGH2 to run the

321 simulation. The model reads the element/connection information (i.e., name and X, Y, and Z

322 coordinates) from the TOUGH2 input file. The outputs are read from the TOUGH2 output file

323 and stored in a 3-dimentional array Results $(m \times n \times h)$ in which $m, n, h$ represent the numbers

324 of elements/connections, printout times and variables. This format allows users to easily analyze

325 the temporal variability of different variables at each element (e.g., temperature, saturation, gas

326 saturation, capillary pressure) and connections (e.g., heat and fluid rates). Users can also observe

327 the spatial variability of these variables at elements at each time in a $2 \mathrm{D}$ slice or in 3D.

328 iMatTOUGH can plot the TOUGH2 output data in both regular and irregular meshes. However,

329 for the irregular mesh, when the vertex coordinates of each element are not available, the shapes

330 of elements cannot be determined and the locations of the elements are specified by the

331 coordinates of the element centers. In the case where the $\mathrm{X}, \mathrm{Y}, \mathrm{Z}$ coordinates of the elements are

332 not provided, iMatTOUGH only plot the temporal variation of the TOUGH2 output at elements.

333 The flux rate flowing into or out of elements can be viewed using flowing vectors or in $\mathrm{X} / \mathrm{Y} / \mathrm{Z}$ 334 direction. 


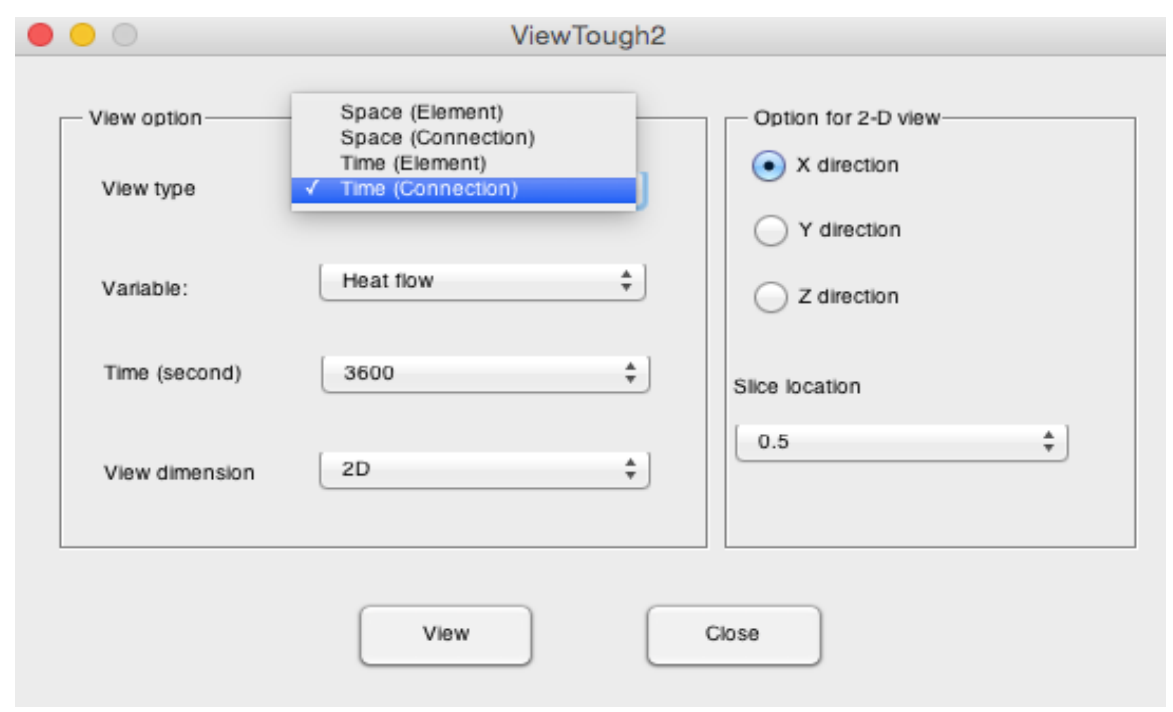

(a)

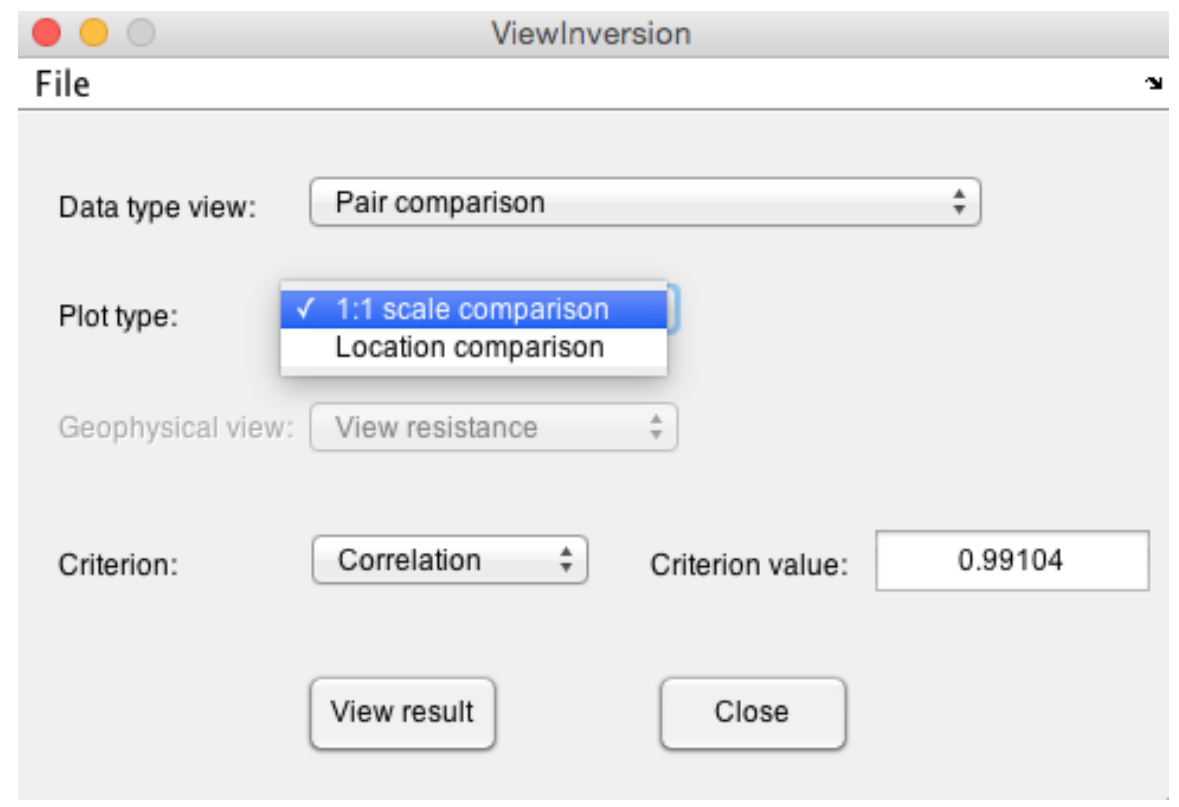

(b)

Figure 6. User interface for viewing (a) TOUGH2 and (b) iTOUGH2 outputs.

\subsection{2. iTOUGH2 viewer}

339 Figure $6 \mathrm{~b}$ presents the GUI for viewing iTOUGH2 outputs. In the iTOUGH2 viewer, users can

340 observe the sensitivity of the model outputs with respect to different parameters at different

341 locations and times, helping them to effectively identify the most influential parameters, 
342 sensitive observations and important measurement times. Also, the program allows easy 343 comparison of measured and modeled data with different chart types, e.g., 1:1 plot or temporal 344 variability of the measured and modeled data at the observation locations. Statistical measures 345 (e.g., correlation coefficient, bias, RMSE) that evaluate the agreement between observation and 346 model results are also shown.

\section{EXAMPLE USE CASE}

349

350

In order to illustrate the capability of iMatTOUGH, we present an example that used TOUGH2 and $\mathrm{iTOUGH} 2$ to estimate the soil hydrological and thermal parameters in vadose zone from matric potential head and temperature data along a transect at the Rifle site, Colorado. We present the pre-processing stage to estimate the initial and boundary conditions and show some outputs generated by iMatTOUGH. Tran et al. (2015) provides an analysis of the use case inversion results. Here, we provide a brief description of the input and output of this case study to illustrate the iMatTOUGH GUI capabilities.

\subsection{Computational mesh and data availability}

The field case study was performed using data from the Berkeley Lab Subsurface Biogeochemical Scientific Focus area study site, located at a floodplain adjoining the Colorado River near Rifle, Colorado (USA). Figure 7a presents the location of this site. The perched aquifer at the site is underlain by low permeability mud- and siltstones of the Eocene Wasatch Formation. Above the Wasatch Formation is a Quaternary alluvial layer consisting of sandy gravely unconsolidated sediments with variable silt and clay contents. The uppermost layer is a silty-clay fill with a thickness of around $1.5-2 \mathrm{~m}$ that was deposited to replace contaminated 
367 soils and sediments removed from the site following reclamation activities related to the site's

368 former history as a uranium mill site. The objective of the inversion is to estimate the soil

369 hydrological and thermal parameters and to quantify the spatiotemporal variability of subsurface

370 soil moisture and temperature, which are crucial information for investigating the biochemical

371 dynamics in Rifle.

Figure $7 \mathrm{~b}$ shows an electrical resistivity image obtained by geophysical inversion using the 374 electrical resistivity tomography (ERT) data to define different soil layers. The three soil layers 375 (fill, alluvium and impermeable Watsch Formation) are well defined and their locations are consistent with the information obtained from borehole lithologs. The X, Y, Z coordinates of the

377 computational domain and boundary between the fill and alluvium layers are extracted from the ERT and put to the iMatTOUGH-MESHMAKER sheet of the EXCEL file (Figure 4) to generate 379 the computational mesh. Figure 7c presents the computational mesh generated by iMatTOUGH. 380 The selected computational domain is a rectangle with $30 \mathrm{~m}$ in width and $6.5 \mathrm{~m}$ in depth. It was 381 divided into 29 equally spaced columns, each with a size of $1 \mathrm{~m}$ in the horizontal direction. In the 382 vertical direction, the cell size is $0.05 \mathrm{~m}$ for the uppermost $2 \mathrm{~m}, 0.3 \mathrm{~m}$ for the next $1.5 \mathrm{~m}$ and 0.6 $383 \mathrm{~m}$ for the last $3 \mathrm{~m}$.

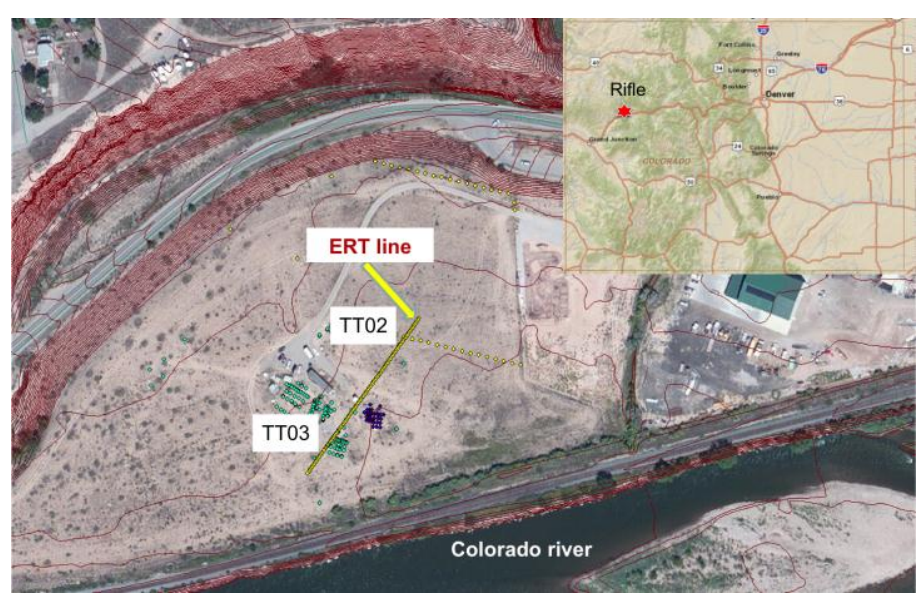

(a) 


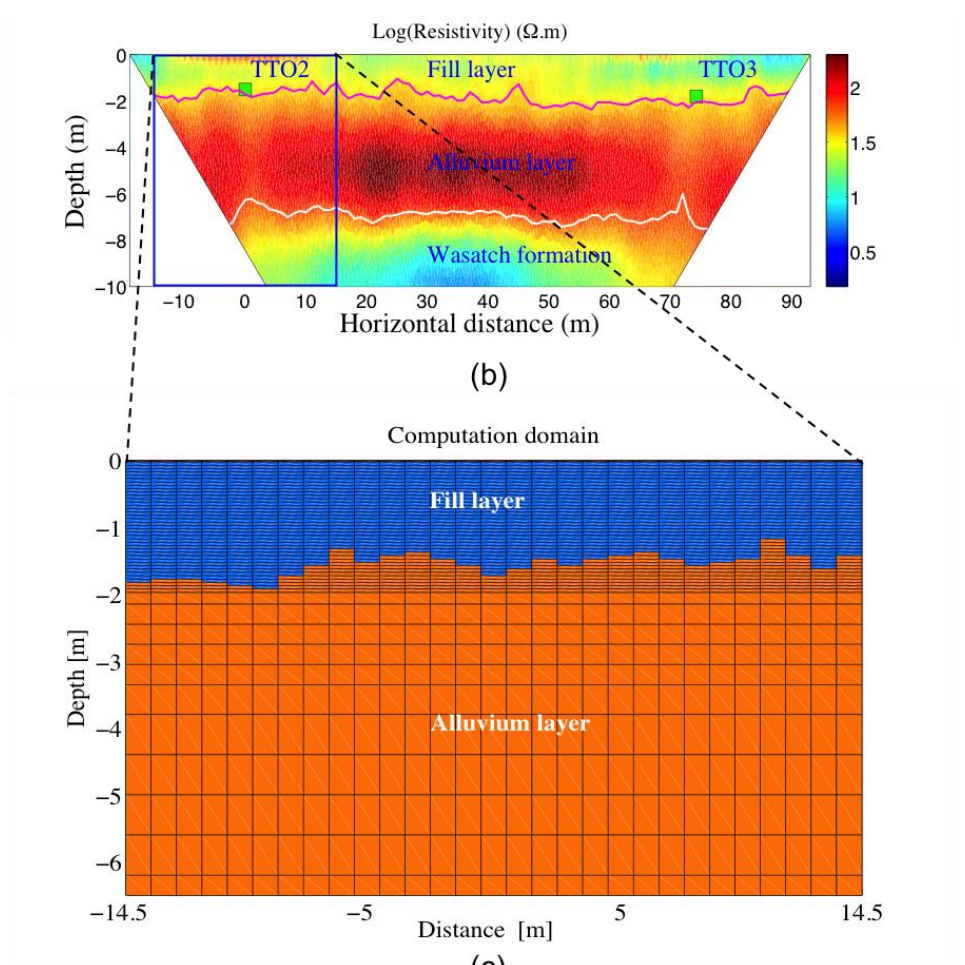

(c)

384 Figure 7. (a) Plan view of the study site in Rifle floodplain of the Colorado River, Colorado and location of the TT02 and TT03 wells and ERT line. (b) The 2-D ERT image obtained by 386 inverting electrical resistivity data. Green square markers denote the fill-alluvium boundary 387 determined from the well logs of TT02 and TT03. The blue rectangular box indicates the hydrological-thermal computational domain. (c) Computational domain for the hydrologicalthermal inversion with associated grid generated by iMatTOUGH.

391 For estimating the hydrological and thermal parameters of both alluvium and fill layers using 392 iTOUGH2, we used the matric potential and temperature over the period from May 4, 2013 to 393 November 25, 2013. The temperature measurements were implemented at six depths $z=0.75,1$, 394 1.5, 2.5, 4.6 and $6 \mathrm{~m}$ below ground surface at the TT03 well. The matric head was occasionally 395 measured using tensiometers at well TTO2 at depths $\mathrm{z}=0.5,1,1.5,2,2.5$ and $3 \mathrm{~m}$. 


\subsection{Initial and boundary conditions}

\subsubsection{Boundary condition}

To specify initial and boundary conditions in TOUGH2, values for three primary variables must be specified, namely (1) pressure, (2) temperature, and (3) either air mass fraction $\left(X_{\text {air }}\right)$ for single-phase conditions (gas or liquid), or gas saturation $\left(S_{g}\right)$ for two-phase conditions. The upper boundary condition is set to the atmospheric pressure adjusted for elevation above sea level using Equation (1). With the altitude $\mathrm{h}=1619 \mathrm{~m}$, the atmospheric pressure at our study site is $\mathrm{P}_{\mathrm{a}}=8.3 \times 10^{4} \mathrm{~Pa}$. Rainfall data were considered as water sources at the surface elements of the computational mesh. Unit conversion from rainfall to source was performed using Equation (11). Figure 9a presents the rainfall data over the simulation period.

The upper boundary condition of the soil surface temperature was approximated from the atmospheric temperature using the method presented in Zheng et al. (1993). We constructed the regression equation between the smoothed daily atmospheric and non-smoothed soil surface temperature using measured data from April 7, 2014 to March 25, 2015 at a location near the study site (reference location). Based on this regression equation and snowpack information, the soil surface temperature can be estimated using Equation (2) or (3). Figure 8 compares the air and estimated soil surface temperature with the measured soil surface temperature at the reference location. The figure indicates that the estimated soil surface temperature much better agrees with the measured one (correlation coefficient $=0.98$, RMSE $=1.62$ ) than the air temperature (correlation coefficient $=0.93, \mathrm{RMSE}=3.2$ ). Assuming that the regression air-soil surface temperature relationship at the reference location is also valid for the study transect and stable in time, we applied it to estimate the top temperature boundary condition from the air 
421 temperature. The estimated surface temperature is shown in the middle panel of Figure 9c.

423 Figure 9d shows the air mass fraction estimated from the atmospheric temperature and humidity 424 data using Equations 4 - 7. Figure 9e presents the bottom pressure boundary condition. As the 425 bottom of the computational domain is situated below the groundwater table, the bottom pressure 426 boundary condition was estimated from groundwater table data using the hydrostatic Equation 427 (8). The bottom temperature condition was approximated from the temperature data measured at 428 depth $z=6 \mathrm{~m}$ using the analytical solution of the heat transport equation (Equation (9)). Finally, 429 the bottom boundary condition of the air mass fraction was a very small value. We subjectively $430 \quad$ set it at $X_{\text {air }}=1.6 \times 10^{-9}$.

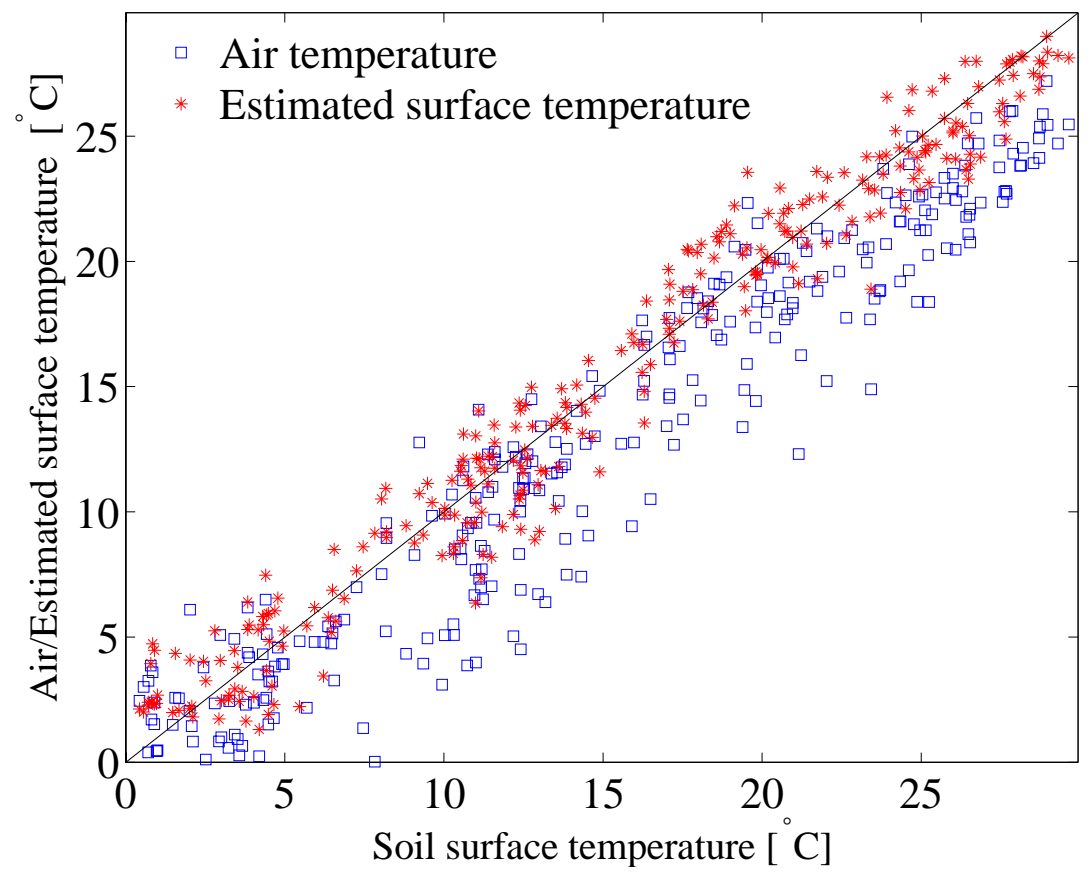

432 Figure 8. Comparison of the daily air temperature and estimated soil surface temperature with the measured soil surface temperature at the reference location. 


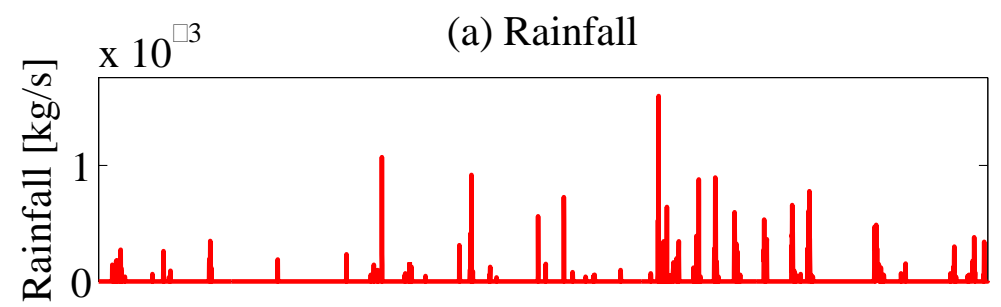

(b) Top boundary temperature

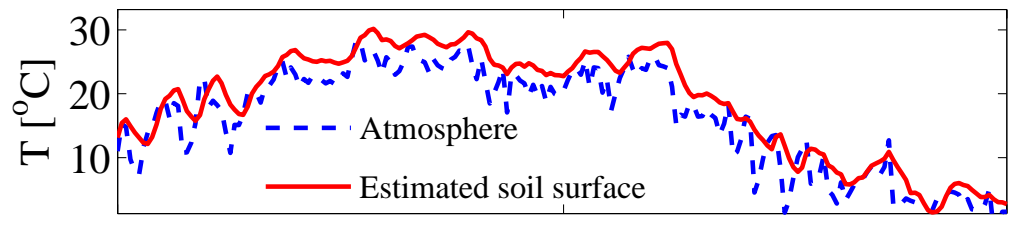

(c) Air mass fraction
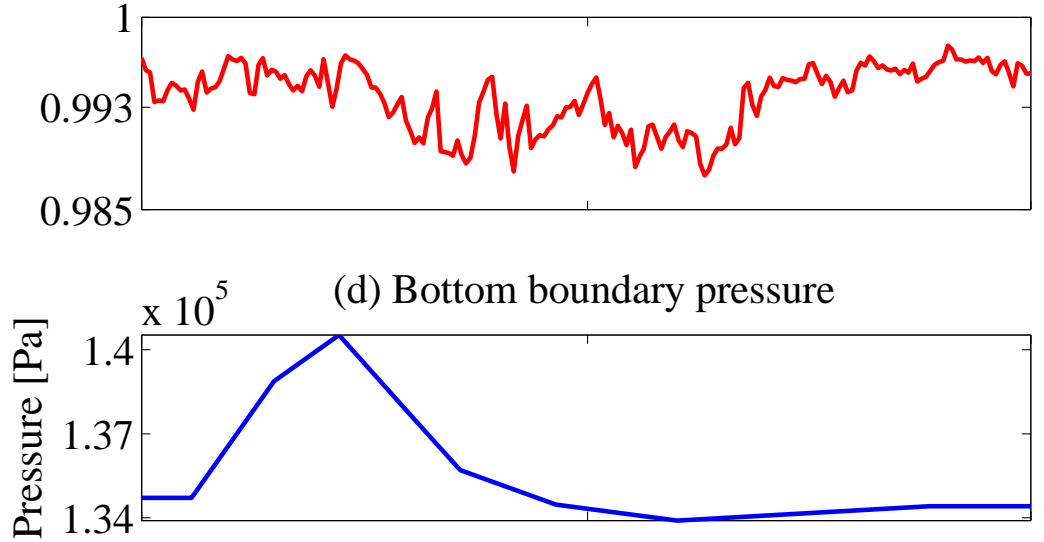

(e) Bottom boundary temperature

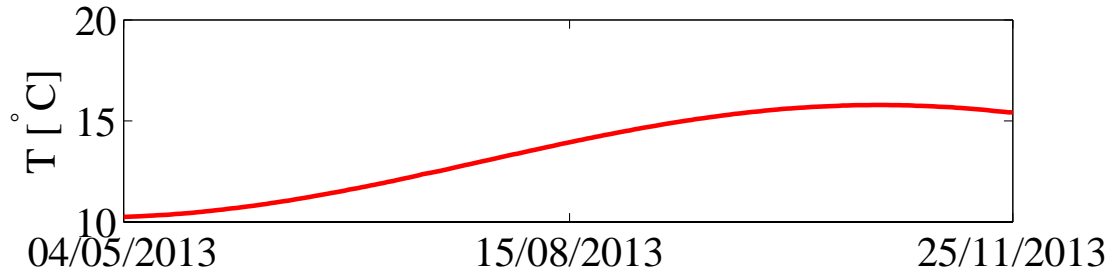

Figure 9. Top and bottom boundary conditions: Rainfall (a), soil surface temperature (b), air mass fraction (c), bottom pressure (d) and bottom temperature (e).

\subsubsection{Initial conditions}

TOUGH2 requires the initial conditions at all elements of the computational domain. Figure 10

441 plots the initial conditions that we used in this study. We refer to section 2.2.4 for the methods to 
442 calculate these initial conditions. The initial temperature profile was obtained by interpolating 443 and extrapolating the temperature measured at depths $0.75,1,1.5,2.5,4.63$ and $6 \mathrm{~m}$ at the 444 beginning of the simulation period. It is worth noting that in TOUGH2 the second initial variable 445 at the saturated zone is $S_{g}$ because a two-phase condition exists, while the second variable is the 446 air mass fraction $\left(X_{\text {air }}\right)$ below the groundwater table (single-phase liquid) and in the atmospheric 447 layer (single-phase gas). It is also noted that the initial liquid saturation of the fill and alluvium 448 layers are significantly different because there is a large discrepancy in their porosities (the 449 porosities are, respectively, equal to 0.4 and 0.2 for the fill and alluvium layers).

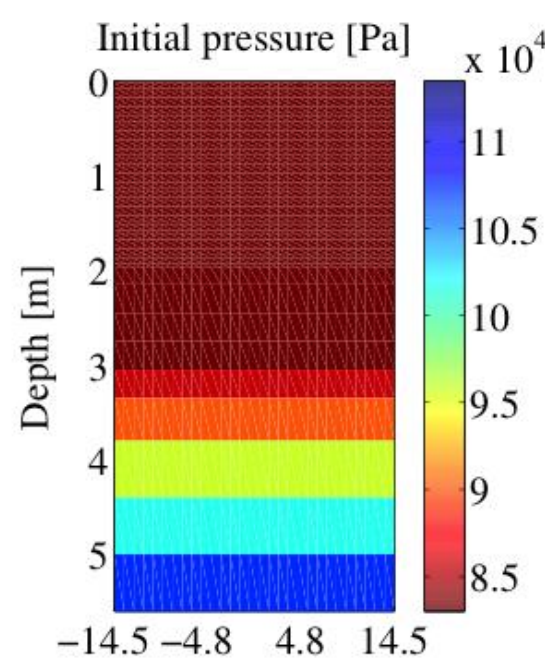

Figure 10. Initial conditions of pressure, gas saturation and temperature for nonisothermal 453 hydrological simulation.

\subsection{Visualization of TOUGH2 and iTOUGH2 outputs}

457 Figure 11 shows examples of the TOUGH2 outputs that were plotted by iMatTOUGH. Figure 458 11a compares the temporal variation of the temperature at several elements of the first column of 
the computational domain $(X=0.5, Y=-14.5 \mathrm{~m})$, namely (A11_1: $Z=-0.025 \mathrm{~m}$, A1D1 1: $Z=-0.65 \mathrm{~m}$, A1f $1, Z=-2.15 \mathrm{~m}$ and A1o $1: Z=-6.2 \mathrm{~m}$ ) over the simulation period. This plot supports users to compare the variation of the soil temperature at different locations and qualitatively analyze the reason for differences in the temporal variations of variables at different locations. For example, Figure 11a indicates that the temporal variation of the soil temperature is more stable at the deep element than the top element due to heat retention effect. The peak of the soil temperature at deeper element also appears later than that at top element because heat takes time to move down. Figure $11 \mathrm{~b}$ shows the liquid water of the whole computational domain at time $t=112$ days in 3-D. Figures $11 \mathrm{c}$ and $\mathrm{d}$, respectively, show the heat flow that comes from each element in the computational domain to its neighbor elements in $Y$ and $Z$ directions at time $t=112$ days. These two figures indicate that the heat flow in the vertical direction is much stronger than that in the horizontal direction. The heat flow in the vertical direction reduces with increasing soil depth.

Figure 12 shows examples of the spatial and temporal variations of the sensitivity coefficients plotted by iMatTOUGH. Figure 12a shows the temporal variation of the sensitivity coefficients of the thermal conductivity of the alluvium and fill layers with respect to the soil temperature at $1 \mathrm{~m}$. This figure allows users to identify the time that the temperature is the most sensitive with respect to thermal conductivity. The figure also helps to compare the influence of different parameters on observation data. Figure $12 \mathrm{~b}$ presents the total sensitivity coefficient of all parameters over the simulation period at different locations. This figure identifies the locations having high sensitivity, which can be used to guide subsequent experimental design. Figure 12c compares the measured and modeled temperature data in a 1:1 plot. Figure $12 \mathrm{~d}$ compares the temporal variation of the modeled and measured temperature at a depth of $1 \mathrm{~m}$. 


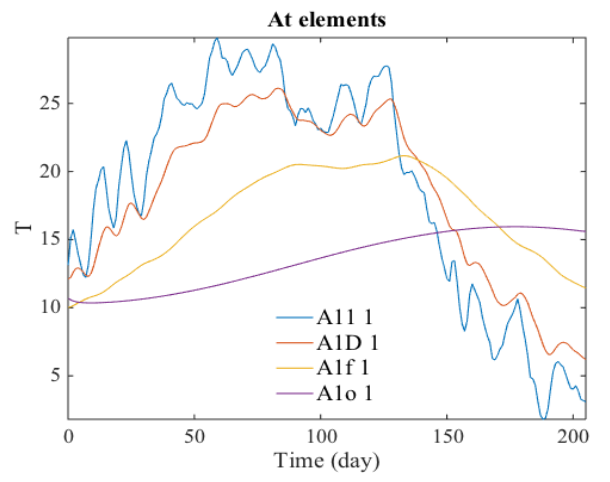

(a)

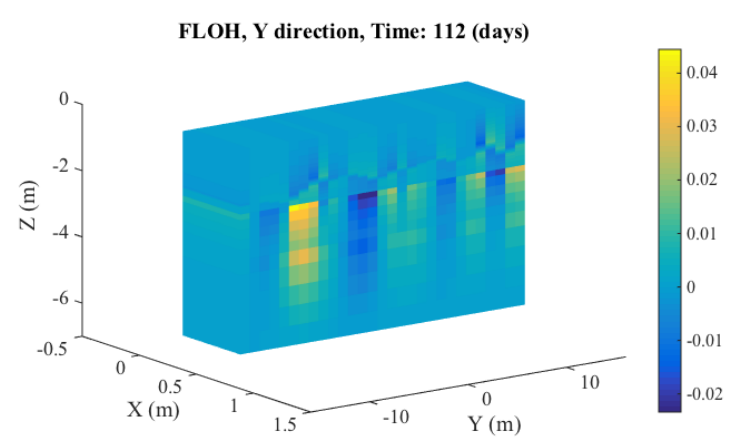

(c)

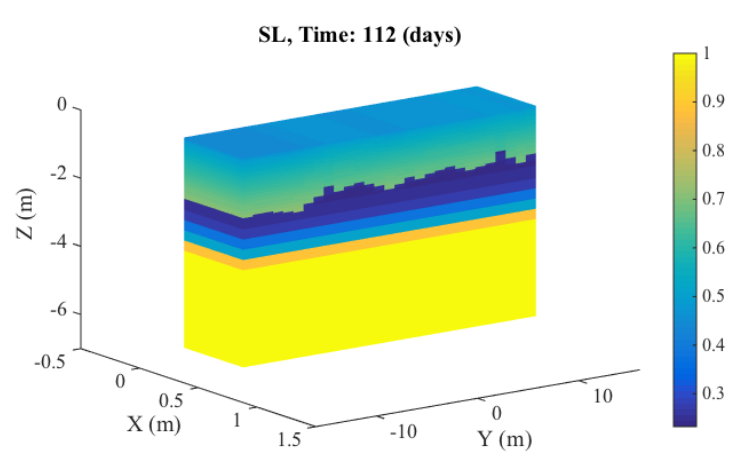

(b)

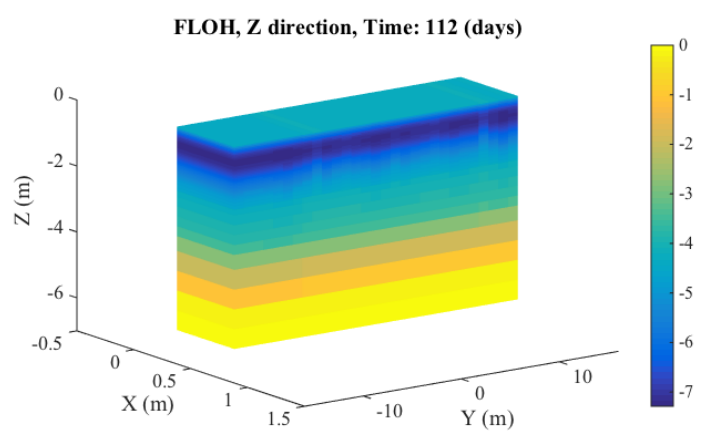

(d)

Figure 11. Examples of TOUGH2 outputs plotted by iMatTOUGH: (a) Temporal variation of the temperature at different elements of the first soil column of the computational domain $(X=$ $0.5, Y=-14.5 \mathrm{~m}$ ), namely (A11_1: $Z=-0.025 \mathrm{~m}$, A1D1 $1: Z=-0.65 \mathrm{~m}$, A1f $1, Z=$ $-2.15 \mathrm{~m}$ and A1o 1: $Z=-6.2 \mathrm{~m}$ ); (b) 3-D subsurface liquid saturation at time $t=112$ days; (c) 3-D heat flow in $Y$ direction at time $t=112$ days; (d) 3-D heat flow in $Z$ direction at time $t=112$ days. 


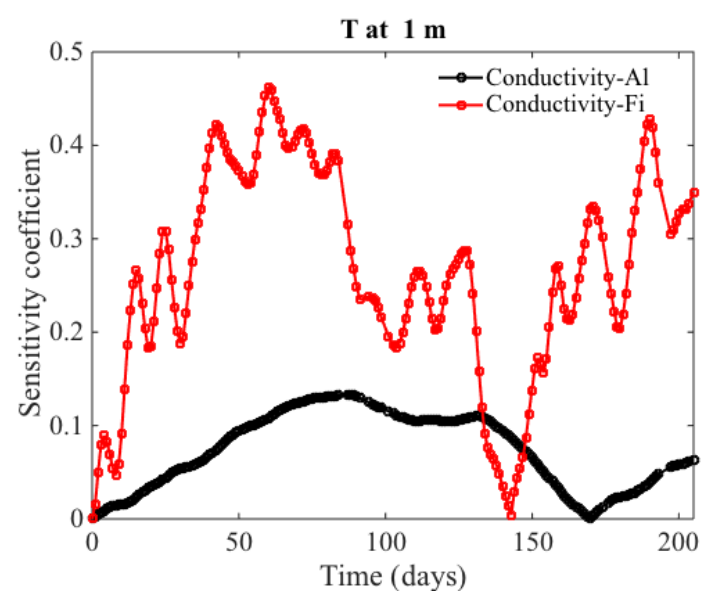

(a)

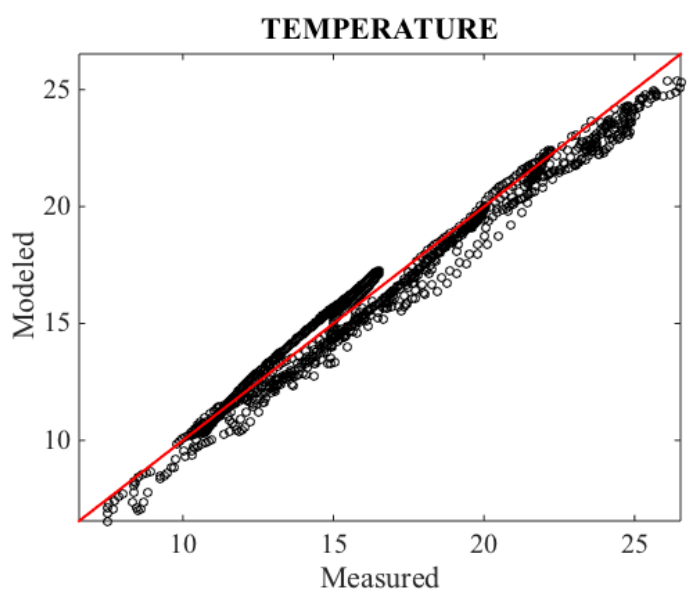

(c)

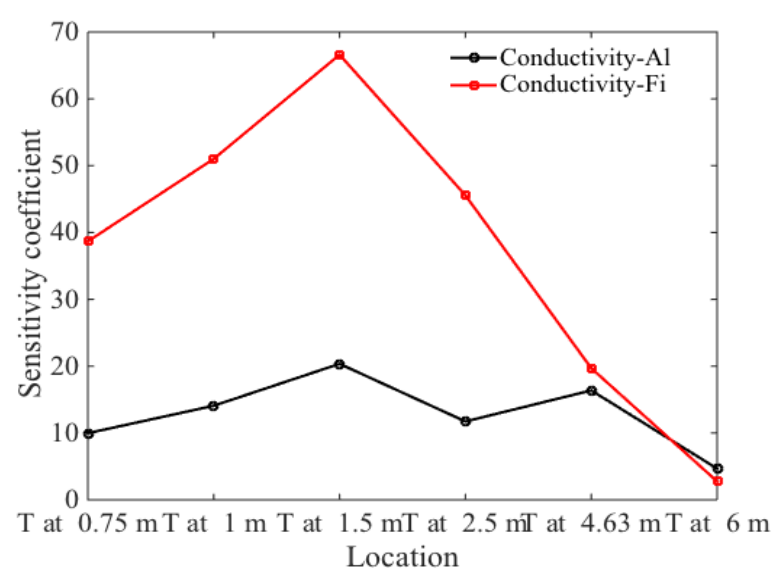

(b)

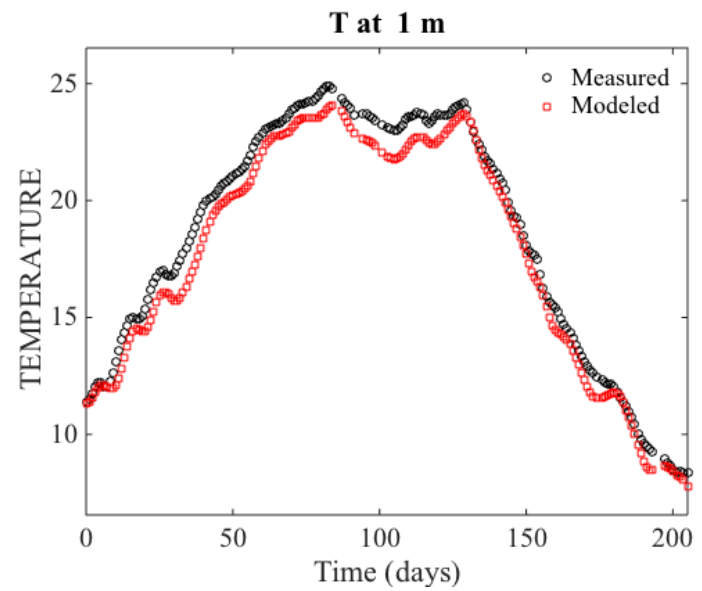

(d)

Figure 12. Examples of iTOUGH2 output plotted by iMatTOUGH: (a) Temporal and (b) spatial variation of the sensitivity coefficients of the temperature data with respect to the thermal conductivity of the alluvium (Al, black) and fill layer (Fi, red); (c) Comparison of the measured and modeled temperature data in 1:1 plot, and (d) their temporal variation at depth $\mathrm{z}=1 \mathrm{~m}$. 


\section{CONCLUSION AND DISCUSSION}

490

iMatTOUGH is a new, Matlab-based tool that allows convenient pre- and post-processing TOUGH2 and iTOUGH2 input and output files. The pre-processing supports users to more easily generate the TOUGH2 and iTOUGH2 input files. The post-processing part helps users to visualize and analyze the TOUGH2 and iTOUGH2 outputs with different types of charts. For its open source code, it is easy for users to update and/or modify the code for their specific purposes or when there are modifications/updates in TOUGH2 and iTOUGH2. The pre-processing of mesh generation, initial and boundary conditions as well as visualization of output data not only are used for TOUGH2 and iTOUGH2 but also can be adapted to other hydrological modeling forward and inversion codes. The tool requires two popular software tools, namely, Matlab and Microsoft Excel to perform all of its tasks. Interested users can download this tool at https://www.dropbox.com/sh/n3yo418puv6ocax/AADTAixV6MS1uH3w0u_541LVa?dl=0

502

The program supports the construction of the rectangular computational mesh and determination of the initial and boundary conditions for the subsurface heat and liquid flow simulation. In the next version, we will demonstrate on integrating the irregular mesh making code (e.g., Berry et al., 2014) into iMatTOUGH to generate the unstructured computational mesh. We also plan to expand the initial and boundary condition processing part so that the program can process the 508 initial and boundary conditions for different application fields (e.g., geothermal, nuclear waste, 509 nutrient cycle and oil \& gas). 


\section{ACKNOWLEDGMENT}

513

514

515

516

517

This material is based upon work supported as part of the Subsurface Science Scientific Focus Area funded by the U.S. Department of Energy, Office of Science, Office of Biological and Environmental Research under Award Number DE-AC02-05CH11231. The authors would like to thank Stefan Finsterle for reading and providing useful suggestions to improve the manuscript.

\section{REFERENCES}

1. Berry, P., S. Bonduá, V. Bortolotti, C. Cormio, E.M. Vasini, 2014. A GIS-based open source pre-processor for georesources numerical modeling, Environmental Modelling \& Software 62, 52-64.

2. Finsterle, S. (1999), iTOUGH2 User's Guide, Lawrence Berkeley National Laboratory, Berkeley, CA.

3. Li, Y., Niewiadomski, M., Trujillo, E., Sunkavalli, S.P., 2011. Tougher: a user-friendly graphical interface for TOUGHREACT. Computers \& Geosciences 37 (6), 775-782.

4. Pruess, K., C. Oldenburg, and G. Moridis (1999), TOUGH2 user's guide, version 2.0, Lawrence Berkeley National Laboratory, Berkeley, CA.

5. Tran, A.P., Dafflon, B., Hubbard, S.S., Kowalsky, M.B., Long, Ph., Tokunaga, T. and Williams, K.H., 2015. Quantifying Shallow Subsurface Water and Heat Dynamics using Coupled Hydrological-thermal-geophysical Inversion. Submitted to Water Resources Research. 
6. Wellmann, J. F., A. Croucher, and K. Regenauer-Lieb, 2012. Python scripting libraries for subsurface fluid and heat flow simulations with TOUGH2 and SHEMAT." Computers \& Geosciences 43, 197-206.

7. Zheng, D., J. E. R. Hunt, and S.W. Running (1993), A daily soil temperature model based on air temperature and precipitation for continental applications, Climate Research, 2, 183-191.

8. van Genuchten, M. T. (1980), A closed-form equation for predicting the hydraulic conductivity of unsaturated soils, Soil Science Society of America Journal, 44, 892-898.

9. van Wijk, W. R., and D. A. de Vries (1963), Periodic temperature variations in homogeneous soils, in Physics of Plant Environment, edited by W. R. van Wijk, pp. 102143, North-Holland Publishing Co., Amsterdam. 